\title{
Single antigen flow beads for identification of human leukocyte antigen antibody specificities in hypersensitized patients with chronic renal failure
}

\author{
MUSTAFA SOYÖZZ ${ }^{1,2}$, TÜLAY KILIÇASLAN-AYNA ${ }^{1,2}$, ASLI ÖZKIZILCIK-KOÇYIĞIT ${ }^{1,2}$, \\ DERYA GÜLEÇ ${ }^{2}$ IBRRAHIM PIRIM ${ }^{1,2}$
}

${ }^{1}$ Department of Medical Biology and Genetics, Faculty of Medicine, İzmir Katip Çelebi University, İzmir, Turkey ${ }^{2}$ Tissue Typing Laboratory, Izmir Tepecik Education and Research Hospital, İzmir, Turkey

\begin{abstract}
Aims of this study were to identify class I and class II antibodies in highly sensitized patients by flow cytometry single antigen bead (FC-SAB) assay and to evaluate according to donor HLA type in order to increase their kidney transplantation chance.

Material and methods: We analyzed 60 hypersensitive patients of 351 individuals, who applied to our laboratory for PRA test in November 2013-December 2014. Flow cytometric PRA screening and single antigen bead commercial kits were used for these analyses.

Results: In our study group, 19 (31.7\%) of these patients were male while 41 (68.3\%) patients were female. The most common acceptable antigens were $A * 02$ (10.11\%), HLA-A*23 (10.11\%), HLA-B*38 $(8.79 \%)$ and $H L A-D R B 1 * 03(7.83 \%)$ in hypersensitive patients. The highest antibody reactivity on $S A B$ was observed against HLA-A*25, HLA-B*45, HLA-DRB $1 * 04$ and HLA-DRB $1 * 08$ antigens.

Conclusions: The determination of these acceptable and unacceptable antigens may increase their transplantation chance. Pre-transplant HLA antibody identifications provide prognostic information with respect to the determination of patients who are at increased risk of graft loss.
\end{abstract}

Key words: flow cytometry, DSA, single antigen bead, HLA.

(Cent Eur J Immunol 2016; 41 (1): 93-100)

\section{Introduction}

Anti-human leucocyte antigen (anti-HLA) antibodies produced by patients via blood transfusions, transplantation history and pregnancies are called panel reactive antibody (PRA) [1]. The individuals with $\geq 85 \%$ PRA ratio are referred to hypersensitized. The obvious solution for the transplantation of these hypersensitized patients is to find an HLA-identical donor. However, it is almost impossible since HLA system has extensive polymorphisms. There are two ways to carry out transplantation of these patients: i) removal of the antibodies that cause the positive crossmatch by various methods used in clinics, ii) definition of the holes in their antibody repertoire to enhance selection of crossmatch-negative HLA mismatched donors [2].

One-third of the patients in United Network for Organ Sharing (UNOS) waiting list are sensitive to HLAs. When the PRA results and waiting duration of the patients on the list were evaluated, it was found that the patients with
$<30 \%$ PRA had kidney transplantation after 493 days, while the patients with $\geq 30 \%$ PRA waited an average of 1047 days for the transplantation [3]. According to these data, it was considered that higher PRA ratio increased the waiting duration in the list. Thus, it decreased the transplantation chance of sensitized ( $\geq 30 \%$ PRA) patients.

Patel and Terasaki [4] demonstrated the impact of complement-dependent lymphocytotoxic cross-match (CDC-XM) test in identifying immunologic risk in renal transplantation. This became the gold standard method for graft allocation and it is still used before transplantations. However, it is well-known that it cannot identify specifically the preexisting donor-specific HLA antibodies (HLADSA) any more (i.e. HLA-A*24). Recently, HLA antibody detection techniques have become more sensitive and specific with solid-phase assays [5]. One of these tests is based on analyzing of patient sera with the beads covered by a single HLA antigen (single antigen bead-SAB) [6]. The clinical significance of the specific antibodies detect-

Correspondence: Mustafa Soyöz, Department of Medical Biology and Genetics, Faculty of Medicine, İzmir Katip Çelebi University,

Aydınlıkevler mah. Cem Meriç Cad. 6780 sok No: 48 Çiğli, 35640 İzmir, Turkey, e-mail: mustafa.soyoz@ikc.edu.tr

Submitted: 18.05.2015; Accepted: 20.07.2015 
ed by these more sensitive techniques has not been fully evaluated for graft survival and definition of acceptable grafts $[5,7]$.

Flow cytometry-based solid phase assays (flowbeads) have at least similar sensitivity for the detection of specific HLA as flow cytometer crossmatch (FCXM) [8]. Therefore, HLA-DSAs can be determined without performing a FCXM test by comparing the HLA antibody specificities of the recipient with HLA-typing of the donor (i.e. virtual XM). The absence or presence of DSA can be decided by virtual XM and it may become invaluable tool for the evaluation of organ allocation and pretransplant risk level [9].

HLA-DSAs produced in recipients are specific to the epitopes of HLA antigens in donors. Thus, these epitopes and determination of acceptable antigens should be considered during the assessment of donor-recipient compatibility. One of the important assessment strategies is use of Acceptable Mismatch program that is used in Eurotransplant. In this strategy, selection of specific panel cells is used during the screening of the serum for each hypersensitized patient and this specific panel cells have only one HLA-A or HLA-B mismatch with the patient. Accordingly, the HLA antigens that the patient can accept may be found by these acceptable mismatches [12].

The aims of the study were to identify class I and class II antibodies in highly sensitized patients by flow cytometry single antigen bead (FC-SAB) assay and to evaluate according to donor HLA type in order to increase their kidney transplantation chance.

\section{Material and methods}

\section{Patients}

In this study, 60 hypersensitive patients of 351 individuals who applied to our laboratory for PRA test in November 2013-December 2014, were included. The patients with $>85 \%$ PRA reactivity were accepted as hypersensitive and tested by FC-SAB. HLA typing of hypersensitive patients were performed by molecular method. Nineteen $(31.7 \%)$ of these patients were male while $41(68.3 \%)$ patients were female. Thirty-three (55\%), 17 (28\%) and $10(17 \%)$ patients were analyzed for Class I, II and both of them by FC-SAB method, respectively. The study was approved by the Committee on Medical Ethics of the Izmir Katip Celebi University Faculty of Medicine.

\section{HLA typing}

Some of the patients were typed for HLA-A, -B and -DR loci by Luminex technology using commercial sequence-specific oligonucleotide (SSO) kits (Gen-Probe, Stanford, USA). The manufacturer's instructions were followed during the procedure. Some of the patients were typed for HLA-A, -B and -DR by serological methods. Some of them were also analyzed in other centers.

\section{Antibody detection}

Flow cytometric PRA screening and single antigen bead commercial kits (One Lambda, Inc.) were used for these analyses according to the protocol recommended by the manufacturer. These beads are used in order to research antibodies against common HLA antigens in our population. $20 \mu \mathrm{l}$ of patient serum was incubated with $5 \mu \mathrm{l}$ of class I- and class II-coated microparticles for $30 \mathrm{~min}$ utes and after incubation the tubes were washed two times by adding $1 \mathrm{ml}$ wash buffer and centrifuging at $1900 \mathrm{rpm}$ for 10 minutes. After the washing step, $100 \mu \mathrm{l}$ fluorescein isothiocyanate-conjugated anti-human IgG (FITC-anti-Ig; Fc fragment specific, Dako, Glostrup, Denmark) was added into the tubes and incubated at room temperature for 30 minutes in the dark. After incubation the washing steps were repeated as mentioned above. The fluorescence of the samples was then assessed by flow cytometer instrument. Positive samples were analyzed for single antigen specific antibodies by using single antigen beads coated with a single HLA antigen. Fluorescence of 5000 events was analyzed on a flow cytometer instrument (BD Facs Calibur, USA).

$\mathrm{SAB}$ analysis was based on excitation and emission of the beads at $488 \mathrm{~nm}$ and $580 \mathrm{~nm}$, respectively. This fluorescence was collected on the FL2 channel on a flow cytometer. The class I bead contained four different groups of beads each containing nine different beads and having a unique FL2 channel shift. Thus, these beads could be separated from each other by the FL2 channel of a flow cytometer. However, the positive reactions of HLA antibodies were detected by the FL1 channel of a cytometer. The main population of beads was gated on the SSC vs. FSC dot blot and FL2 vs. FL1 dot plot was obtained on the gated beads. Gates were set for each bead population that had reacted with the negative control serum on the FL2 vs. FL1 dot plot. The same gates were also used to analyze all the testing sera on their FL2 vs. FL1 dot plot. The reaction was accepted as positive by a shift of the beads to the right of the gate. The channel shift of the beads was then scored and the scores $\geq 4$ were accepted as positive.

\section{Statistical method}

We analyzed HLA allele frequencies in our donor population for hematopoietic stem cell transplantation (HSCT) (2975 donors) and our study group (60 patients) in order to investigate relation between our SAB results and the frequency of HLA alleles.

The comparisons of antigen proportions (as percentages) between the patients groups and the given population proportions were performed using $\mathrm{Z}$ tests and any resulting value such as $Z \geq 1.96$ was considered as statistically significant. 
For comparing proportions, the computation formula given below was utilized:

$$
\begin{gathered}
Z=\frac{\left(\hat{p}_{1}-\hat{p}_{2}\right)-0}{\sqrt{\hat{p}(1-\hat{p})\left(\frac{1}{n_{1}}+\frac{1}{n_{2}}\right)}} \\
\text { were } \hat{p}=\frac{n_{1}+n_{2}}{N_{1}+N_{2}} \text { and } p<0.05 \text { if } Z \geq 1.96
\end{gathered}
$$

\section{Results}

Three hundred and fifty one patients applied to our laboratory for PRA test in November 2013-December 2014. One hundred and twelve (32\%) of these patients were PRA positive (in terms of Class I and/or Class II). Thirty-four $(9.5 \%)$ of these patients were class I positive and class II negative, while $33(9.5 \%)$ were class I negative and class II positive. Forty-five (13.0\%) of these patients were positive for both of the classes. Among these patients, 60 hypersensitive individuals (with $>85 \% \mathrm{PRA}$ ) were analyzed by FCSAB method. The characteristics (Table 1) and FC-SAB results of the patients (Table 2 ) were summarized in tables.

When 60 hypersensitive patients were evaluated in terms of alloimmunization rates, $78 \%, 40 \%$ and $38.3 \%$ were found to have blood transfusion, pregnancy and rejection history, respectively.

According to our FC-SAB results, the most common acceptable antigens were HLA-A*02 (10.11\%), HLA-A*23 (10.11\%), HLA-B*38 (8.79\%), HLADRB $1 * 03(7.83 \%)$. The highest unacceptable antigens on SAB was observed against HLA-A*25, HLA-B*45, HLADRB $1 * 04$ and HLA-DRB $1 * 08$.

The most common alleles were HLA-A*02 $(22 \%)$, HLA-B*35 (20.88\%) and HLA-DRB $1 * 11$ (19.75\%) in HSCT-donor population. In addition, HLA-A*02, HLA-B $* 35$ and HLA-DRB $1 * 11$ frequencies in our study group were $35 \%, 30 \%$ and $30 \%$, respectively. HLA-A $* 25$, HLA-B*45, HLA-DRB $1 * 04$ and HLA-DRB $1 * 08$ frequencies in HSCT-donor population were $0.80 \%, 0.21 \%$, $14.45 \%$ and $1.89 \%$, respectively (Table 3 ).

\section{Discussion}

Kidney transplantation is the good option for the patients with end-stage renal disease [13]. The significance of anti-HLA antibodies in kidney transplantation, especially HLA-DSA, is non-negligible because these antibodies may lead to graft failure after transplantation. In recent years, various methods have been developed to determine these antibodies in order to extend the graft survival. However, even solid-phase PRA screening and specific assays have been insufficient for the identification of the HLA-DSA specifically (i.e. HLA-A24) until recently.

Hypersensitive patients may develop antibodies against a large variety of HLA antigens because of different allo- immunization ways such as pregnancy, blood transfusion, and transplantation. Therefore, these patients have the least chance to receive a suitable organ by standard procedures of the various organ exchange organizations. If special preventive actions are not taken, the number of these patients in the waiting list will increase due to their long waiting duration [14].

In our study group, $23(38.3 \%)$ hypersensitive patients had transplantation history. These patients may become sensitized against the mismatched HLA antigens of the rejected organ. The sensitization incidence after a failed transplant depends on the number of HLA mismatches of the donor. According to Doxiadis et al., it can vary between $20 \%$ (0-1 mismatches) and 46-52\% (5-6 mismatches). Reduction of the HLA mismatches in previous transplants will reduce the incidence of sensitization [2]. However, we did not match functional epitopes of patients and donors.

We also investigated the relation between antibody reactivity on SAB and HLA allele frequencies. We observed that the highest antibody reactivity was against rare alleles [HLA-A*25 (0.80\%), HLA-B*45 (0.21\%), HLADRB $1 * 04(14.45 \%)$ and HLA-DRB $1 * 08(1.89 \%)]$. It was found that the relation between antibody reactivity and HLA allele frequencies was statistically significant because individuals would produce antibodies against rare HLA antigens rather than common antigens since they would also have the common HLA antigens $(p<0.001)$. The only exception in our study was HLA-DRB $1 * 04$. It was one of the antigens against which the highest antibody reactivity was observed, although it has $>10 \%$ frequency in HSCT-donor population. This may be due to allele differences in HSCT-donor population.

Some of our patients seemed as they produced auto-antibodies (Table 2). However, we considered that the results might be due to allele differences or the alterations in three dimensional structure of the antigen during denaturation for the preparation of the beads [15].

In this study, we focused on the method for increasing the chance of hypersensitive patients to find a crossmatch negative donor. It is difficult to determine HLA antibody specifities in highly sensitized patients because these sera include antibodies to many HLA antigens. Moreover, the FC-SAB provides a tool to identify each single antigen reaction against the antibodies in the serum [8]. FC-SAB method can also eliminate undefined results that had been wrongly assigned by computer based programs in specific PRA method. Recently, single antigen bead technology and acceptable mismatch (AM) programs have been used to determine HLA antigens which are suitable for transplantation of hypersensitive patients. Acceptable mismatch programs increase the probability of highly sensitized patients to receive a suitable organ. It was revealed that approximately $60 \%$ of the hypersensitive patients would be transplanted within two years after inclusion in the AM 
Mustafa Soyöz et al.

Table 1. Characteristics of patients

\begin{tabular}{|c|c|c|c|c|c|c|c|c|c|c|c|c|c|c|c|}
\hline $\begin{array}{l}\text { Patient } \\
\text { No }\end{array}$ & Age & Gender & $\begin{array}{c}\text { Number } \\
\text { of blood } \\
\text { transfusion } \\
\text { (Unit) }\end{array}$ & $\mathbf{T x}$ & $\mathbf{A}$ & $\mathbf{C}$ & $\mathbf{P}$ & $\begin{array}{l}\text { Patient } \\
\text { No }\end{array}$ & Age & Gender & $\begin{array}{c}\text { Number } \\
\text { of blood } \\
\text { transfusion } \\
\text { (Unit) }\end{array}$ & $\mathbf{T x}$ & $\mathbf{A}$ & $\mathbf{C}$ & $\mathbf{P}$ \\
\hline 1 & 44 & F & 0 & 0 & 0 & 1 & 6 & 31 & 33 & F & 0 & 0 & 1 & 0 & 1 \\
\hline 2 & 40 & M & 2 & 1 & N/A & N/A & N/A & 32 & 51 & M & 1 & 0 & N/A & N/A & N/A \\
\hline 3 & 67 & $\mathrm{~F}$ & 2 & 0 & 0 & 0 & 5 & 33 & 64 & F & 6 & 0 & 0 & 0 & 0 \\
\hline 4 & 63 & M & 8 & 0 & N/A & N/A & N/A & 34 & 64 & F & 2 & 0 & 0 & 0 & 1 \\
\hline 5 & 42 & M & 1 & 1 & N/A & N/A & N/A & 35 & 54 & F & 0 & 0 & 0 & 1 & 1 \\
\hline 6 & 75 & F & 1 & 1 & 0 & 0 & 8 & 36 & 50 & F & 3 & 0 & 0 & 0 & 0 \\
\hline 7 & 35 & M & 2 & 0 & N/A & N/A & N/A & 37 & 42 & $\mathrm{~F}$ & 0 & 0 & 1 & 0 & 0 \\
\hline 8 & 37 & M & 5 & 1 & N/A & N/A & N/A & 38 & 59 & F & 1 & 1 & 0 & 0 & 0 \\
\hline 9 & 49 & F & 5 & 1 & 1 & 0 & 4 & 39 & 21 & F & 2 & 0 & 0 & 0 & 0 \\
\hline 10 & 16 & F & 1 & 1 & 0 & 0 & 0 & 40 & 65 & $\mathrm{M}$ & 2 & 0 & N/A & N/A & N/A \\
\hline 11 & 25 & $\mathrm{M}$ & 6 & 1 & N/A & N/A & N/A & 41 & 28 & $\mathrm{M}$ & 1 & 0 & N/A & N/A & N/A \\
\hline 12 & 30 & $\mathrm{M}$ & 1 & 1 & N/A & N/A & N/A & 42 & 45 & $\mathrm{~F}$ & 1 & 0 & 0 & 1 & 1 \\
\hline 13 & 43 & F & 3 & 0 & 1 & 1 & 3 & 43 & 64 & $\mathrm{~F}$ & 3 & 0 & 0 & 1 & 8 \\
\hline 14 & 37 & $\mathrm{~F}$ & 1 & 0 & 0 & 0 & 3 & 44 & 62 & $\mathrm{M}$ & 1 & 0 & N/A & N/A & N/A \\
\hline 15 & 38 & F & 1 & 1 & 0 & 1 & 1 & 45 & 60 & $\mathrm{M}$ & 1 & 0 & N/A & N/A & N/A \\
\hline 16 & 46 & F & 1 & 1 & 0 & 0 & 0 & 46 & 22 & F & 1 & 1 & 0 & 0 & 0 \\
\hline 17 & 57 & F & 0 & 0 & 0 & 0 & 0 & 47 & 46 & F & 1 & 1 & 0 & 0 & 0 \\
\hline 18 & 52 & $\mathrm{~F}$ & 5 & 0 & 1 & 0 & 4 & 48 & 35 & F & 0 & 0 & 0 & 1 & 2 \\
\hline 19 & 58 & F & 2 & 0 & 3 & 0 & 2 & 49 & 40 & $\mathrm{~F}$ & 1 & 0 & 0 & 1 & 2 \\
\hline 20 & 31 & F & 1 & 1 & 0 & 0 & 0 & 50 & 45 & F & 2 & 0 & 0 & 0 & 3 \\
\hline 21 & 57 & F & 1 & 0 & 1 & 1 & 6 & 51 & 43 & M & 4 & 1 & N/A & N/A & N/A \\
\hline 22 & 62 & $\mathrm{M}$ & 3 & 1 & N/A & N/A & N/A & 52 & 34 & F & 1 & 0 & 0 & 0 & 1 \\
\hline 23 & 59 & $\mathrm{~F}$ & 4 & 0 & 0 & 0 & 0 & 53 & 51 & $\mathrm{~F}$ & 3 & 0 & 0 & 0 & 1 \\
\hline 24 & 34 & F & 0 & 1 & 0 & 0 & 0 & 54 & 54 & $\mathrm{~F}$ & 0 & 1 & 0 & 0 & 0 \\
\hline 25 & 58 & F & 30 & 0 & 3 & 2 & 10 & 55 & 25 & $\mathrm{~F}$ & 3 & 1 & 0 & 0 & 0 \\
\hline 26 & 39 & $\mathrm{~F}$ & 2 & 0 & 2 & 0 & 3 & 56 & 4 & $M$ & 1 & 1 & N/A & N/A & N/A \\
\hline 27 & 51 & $\mathrm{~F}$ & 0 & 0 & 0 & 1 & 0 & 57 & 37 & $\mathrm{~F}$ & 2 & 1 & 0 & 0 & 0 \\
\hline 28 & 54 & F & 3 & 1 & 0 & 0 & 1 & 58 & 44 & $\mathrm{M}$ & $70-80$ & 0 & N/A & N/A & N/A \\
\hline 29 & 46 & M & 1 & 0 & N/A & N/A & N/A & 59 & 65 & M & 2 & 0 & N/A & N/A & N/A \\
\hline 30 & 40 & $\mathrm{~F}$ & 1 & 0 & 0 & 0 & 1 & 60 & 35 & $\mathrm{M}$ & 0 & 1 & N/A & N/A & N/A \\
\hline
\end{tabular}

$T x$-transplantation; $M$ - male; $F$-female; $A$-abortus; $C$-curettage; $P$ - pregnancy; N/A - not applicable 
Table 2. Comparison of HLA types, PRA and SAB results of the patients

\begin{tabular}{|c|c|c|c|c|}
\hline \multirow{2}{*}{$\begin{array}{l}\text { Patient } \\
\text { No }\end{array}$} & \multirow[t]{2}{*}{ HLA types of the patients } & \multicolumn{2}{|c|}{ PRA\% } & \multirow[t]{2}{*}{ SAB Results ${ }^{\mathrm{a}}$} \\
\hline & & CI & CII & \\
\hline 1 & $\mathrm{~A} * 26, \mathrm{~A} * 68, \mathrm{~B} * 55,-, \mathrm{DRB} 1 * 13,-$ & 94 & 100 & $\begin{array}{c}A * 01, A * 03, A * 29, A * 30, A * 26, A * 68, A * 11, A * 34, \\
A * 33, B * 40, B * 07, B * 55, D R B 1 * 13, D R B 1 * 12 \\
\text { DRB } 1 * 03\end{array}$ \\
\hline 2 & $\mathrm{~A} * 02, \mathrm{~A} * 68, \mathrm{~B} * 27, \mathrm{~B} * 53, \mathrm{DRB} 1 * 11,-$ & 100 & 100 & $\begin{array}{l}\text { A*02, A*68, A*24, B*51, B*13, B*44, B*38, B*57, } \\
\text { B*53, DRB1 } 111, \text { DRB } 1 * 08, \text { DRB } 1 * 13, \text { DRB1*15, } \\
\text { DRB } 1 * 16, \text { DRB } 1 * 03, \text { DRB } 1 * 01, \text { DRB } 1 * 04\end{array}$ \\
\hline 3 & $\mathrm{~A} * 21, \mathrm{~A} * 02, \mathrm{~B} * 37,-, \mathrm{DRB} 1 * 01, \mathrm{DRB} 1 * 10$ & 100 & 100 & $\begin{array}{c}\mathrm{A} * 01, \mathrm{~A} * 02, \mathrm{~B} * 37, \mathrm{DRB} 1 * 01, \mathrm{DRB} 1 * 01, \\
\text { DRB } 1 * 10\end{array}$ \\
\hline 4 & A23(19), A32(19), B52(5), B35, DRB1*15, DRB1*16 & 100 & Neg & $\begin{array}{c}\mathrm{A} * 23, \mathrm{~A} * 32, \mathrm{~B} * 52, \mathrm{~B} * 35, \mathrm{~B} * 51, \mathrm{~B} * 18, \mathrm{~B} * 15, \mathrm{~B} * 45, \\
\mathrm{~B} * 14\end{array}$ \\
\hline 5 & $\mathrm{~A} * 02, \mathrm{~B} * 35, \mathrm{~B} * 51, \mathrm{DRB} 1 * 09, \mathrm{DRB} 1 * 14$ & 100 & 96.67 & $\begin{array}{l}\text { B*18, B*15, B*44, B*52, B*14, DRB } 1 * 09, \\
\text { DRB } 1 * 10, \text { DRB } 1 * 14, \text { DRB } 1 * 17, \text { DRB } 1 * 03\end{array}$ \\
\hline 6 & A2, A24, B8, B61, DR03, DR15 & 94 & Neg & $A * 02, A^{*} 24, B * 08, B * 40$ \\
\hline 7 & $\mathrm{~A} * 24, \mathrm{~A} * 29, \mathrm{~B} * 35,-, \mathrm{DRB} 1 * 15, \mathrm{DRB} 1 * 16$ & Neg & 96.67 & DRB $1 * 01, \mathrm{DRB} 1 * 10, \mathrm{DRB} 1 * 15, \mathrm{DRB} 1 * 16$ \\
\hline 8 & $\mathrm{~A} 1, \mathrm{~A} 3, \mathrm{~B} 7, \mathrm{~B} 60, \mathrm{DRB} 1 * 10, \mathrm{DRB} 1 * 12$ & 100 & 100 & $\begin{array}{c}\text { A*01, A*03, B*07, B*40, DRB } 1 * 01, \text { DRB } 1 * 07 \\
\text { DRB } 1 * 10, \text { DRB } 1 * 12, \text { DRB } 1 * 16, \text { DRB } 1 * 09 \\
\text { DRB } 1 * 15\end{array}$ \\
\hline 9 & $\mathrm{~A} * 11, \mathrm{~A} * 68, \mathrm{~B} * 35,-, \mathrm{DRB} 1 * 01, \mathrm{DRB} 1 * 13$ & 100 & 100 & $\begin{array}{c}\mathrm{A} * 11, \mathrm{~A} * 68, \mathrm{~B} * 35, \mathrm{DRB} 1 * 01, \mathrm{DRB} 1 * 01, \mathrm{DRB} 1 * 12, \\
\text { DRB } 1 * 13, \mathrm{DRB} 1 * 03\end{array}$ \\
\hline 10 & A1, A32(19), B38(16), DRB1*13, DRB $1 * 14$ & 100 & 86.67 & $\begin{array}{c}\text { A*01, A*32, B*38, DRB } 1 * 07, \text { DRB } 1 * 04, \text { DRB } 1 * 13, \\
\text { DRB } 1 * 14, \text { DRB } 1 * 15, \text { DRB } 1 * 16, \text { DRB } 1 * 03, \\
\text { DRB } 1 * 01, \text { DRB } 1 * 09, \text { DRB } 1 * 15\end{array}$ \\
\hline 11 & $\mathrm{~A} * 02, \mathrm{~A} * 03, \mathrm{~B} * 55, \mathrm{DRB} 1 * 04, \mathrm{DRB} 1 * 14$ & 98 & Neg & $\begin{array}{c}\mathrm{A} * 02, \mathrm{~A} * 03, \mathrm{~B} * 13, \mathrm{~B} * 18, \mathrm{~B} * 35, \mathrm{~B} * 15, \mathrm{~B} * 40, \mathrm{~B} * 8, \\
\mathrm{~B} * 14, \mathrm{~B} * 55\end{array}$ \\
\hline 12 & $\mathrm{~A} 1, \mathrm{~A} 2, \mathrm{~B} 49(21), \mathrm{B} 35, \mathrm{DRB} 1 * 11, \mathrm{DRB} 1 * 13$ & Neg & 96.67 & DRB $1 * 01, \mathrm{DRB} 1 * 04, \mathrm{DRB} 1 * 10, \mathrm{DRB} 1 * 15$ \\
\hline 13 & $\mathrm{~A} * 01, \mathrm{~A} * 11, \mathrm{~B} * 52, \mathrm{~B} * 53, \mathrm{DRB} 1 * 07, \mathrm{DRB} 1 * 10$ & Neg & 93.33 & DRB $1 * 07, \mathrm{DRB} 1 * 10, \mathrm{DRB} 1 * 52, \mathrm{DRB} 1 * 53$, \\
\hline 14 & $\mathrm{~A} * 02, \mathrm{~A} * 03, \mathrm{~B} * 18, \mathrm{~B} * 44, \mathrm{DRB} 1 * 01, \mathrm{DRB} 1 * 04$ & Neg & 93.33 & DRB1*01, DRB $1 * 04$, DRB $1 * 10$, DRB $1 * 15$ \\
\hline 15 & $\mathrm{~A} * 11, \mathrm{~A} * 34, \mathrm{~B} * 18, \mathrm{~B} * 35, \mathrm{DRB} 1 * 13, \mathrm{DRB} 1 * 14$ & 92 & Neg & $\begin{array}{c}A * 03, A^{*} 11, A * 23, A * 24, B * 51, B * 52, B * 18, B * 35, \\
B * 15, B * 38, B * 07, B * 08, B * 14, B * 55\end{array}$ \\
\hline 16 & $\mathrm{~A} * 03, \mathrm{~A} * 32, \mathrm{~B} * 08,-, \mathrm{DRB} 1 * 03, \mathrm{DRB} 1 * 11$ & 92 & Neg & 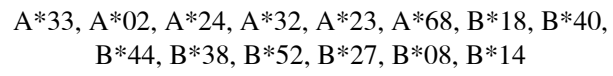 \\
\hline 17 & A26, A30, B51, B13, DRB1*04, DRB $1 * 11$ & 100 & Neg & $\begin{array}{c}A * 26, A * 30, A * 33, A * 34, B * 49, B * 51, B * 52, B * 13, \\
B * 38\end{array}$ \\
\hline 18 & $\mathrm{~A} 1, \mathrm{~A} 11, \mathrm{~B} 44, \mathrm{~B} 49, \mathrm{DRB} 1 * 03, \mathrm{DRB} 1 * 13$ & 96 & Neg & $\begin{array}{c}A * 01, A * 11, A * 23, A * 30, A^{*} 31, B * 13, B * 44, B * 49, \\
B * 57\end{array}$ \\
\hline 19 & A3, B35, B52, DRB1*13, DRB1*14 & 94 & Neg & $\begin{array}{c}A * 03, \mathrm{~B} * 49, \mathrm{~B} * 51, \mathrm{~B} * 13, \mathrm{~B} * 18, \mathrm{~B} * 35, \mathrm{~B} * 15, \mathrm{~B} * 38, \\
\mathrm{~B} * 57, \mathrm{~B} * 14\end{array}$ \\
\hline 20 & $\mathrm{~A} * 23, \mathrm{~A} * 26, \mathrm{~B} * 35, \mathrm{~B} * 50, \mathrm{DRB} 1 * 04, \mathrm{DRB} 1 * 13$ & 100 & Neg & $\begin{array}{c}A * 01, A * 02, A * 25, A * 26, A * 23, A * 30, A * 31, B * 49, \\
B * 35\end{array}$ \\
\hline 21 & $\mathrm{~A} * 02, \mathrm{~A} * 32, \mathrm{~B} * 27, \mathrm{~B} * 41, \mathrm{DRB} 1 * 04, \mathrm{DRB} 1 * 13$ & 100 & Neg & $A * 01, A * 02, A * 23, A * 32, B * 27, B * 40$ \\
\hline 22 & A24(9), A30(19), B13, B18, DR7, DR53 & 97 & 97 & DRB $1 * 02$, DRB $1 * 07$ \\
\hline 23 & $\mathrm{~A} 2, \mathrm{~A} 24, \mathrm{~B} 51, \mathrm{~B} 35, \mathrm{DRB} 1 * 03, \mathrm{DRB} 1 * 13$ & Neg & 90 & $\begin{array}{l}\text { DRB } 1 * 01, \mathrm{DRB} 1 * 10, \mathrm{DRB} 1 * 13, \mathrm{DRB} 1 * 15 \\
\mathrm{DRB} 1 * 16, \mathrm{DRB} 1 * 03, \mathrm{DRB} 1 * 09\end{array}$ \\
\hline 24 & $\begin{array}{c}\text { A29(19), A30(19), B13, B77(15), DRB } 1 * 11 \text {, } \\
\text { DRB } 1 * 14\end{array}$ & 100 & Neg & FM \\
\hline
\end{tabular}


Table 2. Cont.

\begin{tabular}{|c|c|c|c|c|}
\hline 25 & A24, B35, B55, DRB $1 * 04, \mathrm{DRB} 1 * 13$ & 100 & 96 & $\begin{array}{l}\text { DRB } 1 * 11, \mathrm{DRB} 1 * 03, \mathrm{DRB} 1 * 13, \mathrm{DRB} 1 * 14, \\
\text { DRB } 1 * 09, \mathrm{DRB} 1 * 04\end{array}$ \\
\hline 26 & A2, A32(19), B18, B35, DRB1*11 & 96 & 93.33 & $\begin{array}{c}\mathrm{A} * 02, \mathrm{~A} * 29, \mathrm{~A} * 68, \mathrm{~A} * 32, \mathrm{~A} * 33, \mathrm{~B} * 51, \mathrm{~B} * 18, \\
\mathrm{~B} 35, \mathrm{~B} * 15, \mathrm{~B} * 45, \mathrm{~B} * 44, \mathrm{~B} * 38, \mathrm{~B} * 57, \mathrm{~B} * 52, \mathrm{~B} * 14, \\
\mathrm{DRB} 1 * 08, \mathrm{DRB} 1 * 11\end{array}$ \\
\hline 27 & A11, A24, B49, B51, DRB $1 * 08, \mathrm{DRB} 1 * 11$ & Neg & 100 & $\begin{array}{l}\text { DRB } 1 * 08, \text { DRB } 1 * 11, \text { DRB } 1 * 13, \text { DRB } 1 * 16, \\
\text { DRB } 1 * 03, \text { DRB } 1 * 15\end{array}$ \\
\hline 28 & $\mathrm{~A} 24, \mathrm{~A} 26, \mathrm{~B} 27, \mathrm{~B} 40, \mathrm{DRB} 1 * 04, \mathrm{DRB} 1 * 11$ & 88 & $\mathrm{Neg}$ & $\begin{array}{c}A * 25, A^{* 26}, A^{*} 23, A^{*} 24, A^{*} 32, B^{*} 18, B * 40, B * 38 \\
B * 57, B * 27, B * 8, B * 14\end{array}$ \\
\hline 29 & $\mathrm{~A} * 26, \mathrm{~A} * 29, \mathrm{~B} * 38, \mathrm{~B} * 51, \mathrm{DRB} 1 * 03, \mathrm{DRB} 1 * 13$ & Neg & 93.33 & $\begin{array}{c}\mathrm{DRB} 1 * 08, \mathrm{DRB} 1 * 11, \mathrm{DRB} 1 * 13, \mathrm{DRB} 1 * 14 \\
\mathrm{DRB} 1 * 15, \mathrm{DRB} 1 * 16, \mathrm{DRB} 1 * 03, \mathrm{DRB} 1 * 01 \\
\mathrm{DRB} 1 * 12\end{array}$ \\
\hline 30 & $\mathrm{~A} * 02, \mathrm{~A} * 03, \mathrm{~B} * 51,-, \mathrm{DRB} 1 * 04, \mathrm{DRB} 1 * 14$ & 100 & Neg & $\begin{array}{c}A * 02, A^{*} 03, A^{*} 25, A^{*} 29, A * 30, A * 26, A * 68, A * 11, \\
A^{*} 34, A * 32, A * 33, B * 51\end{array}$ \\
\hline 31 & $\mathrm{~A} * 66, \mathrm{~A} * 68, \mathrm{~B} * 18, \mathrm{~B} * 41, \mathrm{DRB} 1 * 11, \mathrm{DRB} 1 * 13$ & 94 & Neg & $\begin{array}{c}A * 66, A * 30, A * 34, A * 33, A * 31, B * 18, B * 41, B * 07, \\
B^{*} 14, B * 55\end{array}$ \\
\hline 32 & $A * 23, A * 24, B * 40, B * 49, D R B 1 * 11, D R B 1 * 15$ & 98 & Neg & $A * 23, A * 24, B * 40, B * 49, B * 27$ \\
\hline 33 & $\mathrm{~A} * 02, \mathrm{~A} * 26, \mathrm{~B} * 08, \mathrm{~B} * 38, \mathrm{DRB} 1 * 03, \mathrm{DRB} 1 * 11$ & 94 & Neg & 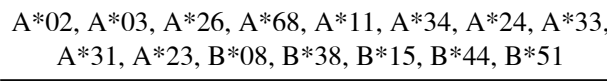 \\
\hline 34 & $\mathrm{~A} * 02, \mathrm{~A} * 03, \mathrm{~B} * 44, \mathrm{~B} * 51, \mathrm{DRB} 1 * 07, \mathrm{DRB} 1 * 10$ & Neg & 96.67 & DRB1*07, DRB1*10, DRB1*01,09, DRB4*01 \\
\hline 35 & $\mathrm{~A} * 31, \mathrm{~A} * 32, \mathrm{~B} * 14, \mathrm{~B} * 15, \mathrm{DRB} 1 * 01, \mathrm{DRB} 1 * 13$ & 100 & Neg & $A * 29, A * 30, A * 31, A * 32, A * 33, B 15, B * 14$ \\
\hline 36 & $\mathrm{~A} * 24, \mathrm{~A} * 26, \mathrm{~B} * 35, \mathrm{~B} * 58, \mathrm{DRB} 1 * 01, \mathrm{DRB} 1 * 13$ & 96 & Neg & $\begin{array}{c}A * 01, A * 25, A * 26, A * 11, A * 23, A * 24, B * 35, B * 40, \\
B * 45, B * 07\end{array}$ \\
\hline 37 & $\mathrm{~A} * 02,-, \mathrm{B} * 27, \mathrm{~B} * 51, \mathrm{DRB} 1 * 08, \mathrm{DRB} 1 * 15$ & 100 & Neg & $\mathrm{A} * 02, \mathrm{~B} * 49, \mathrm{~B} * 51, \mathrm{~B} * 13, \mathrm{~B} * 38, \mathrm{~B} * 52, \mathrm{~B} * 27$ \\
\hline 38 & $\mathrm{~A} * 01, \mathrm{~A} * 23, \mathrm{~B} * 08, \mathrm{~B} * 49, \mathrm{DRB} 1 * 11, \mathrm{DRB} 1 * 13$ & 96 & $\mathrm{Neg}$ & $\begin{array}{c}\mathrm{A} * 01, \mathrm{~A} * 23, \mathrm{~B} * 49, \mathrm{~B} * 13, \mathrm{~B} * 15, \mathrm{~B} * 40, \mathrm{~B} * 44, \mathrm{~B} * 38, \\
\mathrm{~B} * 07, \mathrm{~B} * 27, \mathrm{~B} * 14\end{array}$ \\
\hline 39 & $\mathrm{~A} * 11, \mathrm{~A} * 24, \mathrm{~B} * 51, \mathrm{~B} * 57, \mathrm{DRB} 1 * 04, \mathrm{DRB} 1 * 07$ & 98 & Neg & $\begin{array}{c}A * 01, A^{*} 02, A^{*} 03, A * 11, A * 26, A * 68, A * 33, A * 31, \\
A^{*} 23, A^{*} 25, A^{*} 29, A^{*} 32, B * 51, B * 57\end{array}$ \\
\hline 40 & $\mathrm{~A} * 02, \mathrm{~A} * 32, \mathrm{~B} * 18, \mathrm{~B} * 38, \mathrm{DRB} 1 * 03, \mathrm{DRB} 1 * 04$ & 100 & $\mathrm{Neg}$ & $\begin{array}{c}A * 02, A * 25, A * 29, A * 30, A * 68, A * 11, A * 34, A * 32, \\
A * 33, A * 31, B * 51, B * 18, B * 38, B * 14, B * 08\end{array}$ \\
\hline 41 & A2, A24, B35, B50, DRB $1 * 04$, DRB $1 * 07$ & Neg & 90 & DRB $1 * 07$, DRB $1 * 01$, DRB $1 * 04$ \\
\hline 42 & A2 A24 B35 B50 DRB1*04 DRB1*07 & 100 & Neg & $\mathrm{A} * 01, \mathrm{~B} * 13, \mathrm{~B} * 44, \mathrm{~B} * 57$ \\
\hline 43 & $\mathrm{~A} * 02, \mathrm{~A} * 38, \mathrm{~B} * 44, \mathrm{~B} * 46, \mathrm{DRB} 1 * 11, \mathrm{DRB} 1 * 12$ & 100 & Neg & $A * 02, A * 68$ \\
\hline 44 & A69(28), A30(19), B51(5), B35, DRB $1 * 04, \mathrm{DRB} 1 * 11$ & 96 & $\mathrm{Neg}$ & $\begin{array}{c}A * 69, A * 02, A * 29, A * 30, A * 68, A * 32, A * 33, A * 31, \\
B * 49, B * 51, B * 35, B * 38, B * 55\end{array}$ \\
\hline 45 & $\mathrm{~A} * 01,-, \mathrm{B} * 08,-, \mathrm{DRB} 1 * 03,-$ & 100 & Neg & $\mathrm{A} * 01, \mathrm{~B} * 08$ \\
\hline 46 & $\mathrm{~A} 3, \mathrm{~A} 23, \mathrm{~B} 49, \mathrm{DRB} 1 * 04$ & 98 & Neg & $A * 30, A * 24, A * 03, A * 23, B * 44, B * 49$ \\
\hline 47 & $\mathrm{~A} * 33, \mathrm{~A} * 69, \mathrm{~B} * 49, \mathrm{~B} * 51, \mathrm{DRB} 1 * 04, \mathrm{DRB} 1 * 11$ & 92 & Neg & $\begin{array}{c}A * 33, A^{*} 02, A * 24, A * 32, A * 23, A * 68, B * 18, B * 40, \\
B * 44, B * 38, B * 52, B * 27, B * 08, B * 14\end{array}$ \\
\hline 48 & $\mathrm{~A} * 02, \mathrm{~A} * 32, \mathrm{~B} * 44, \mathrm{~B} * 55, \mathrm{DRB} 1 * 03,-$ & Neg & 97 & $\mathrm{DRB} 1 * 03$ \\
\hline 49 & A3, B51(15), B50(21), DRB $1 * 01, \mathrm{DRB} 1 * 07$ & 94 & $\mathrm{Neg}$ & $\begin{array}{c}\mathrm{A} * 03, \mathrm{~B} * 50, \mathrm{~B} * 51, \mathrm{~B} * 49, \mathrm{~B} * 15, \mathrm{~B} * 40, \mathrm{~B} * 44, \mathrm{~B} * 55, \\
\mathrm{DRB} 1 * 07\end{array}$ \\
\hline 50 & $\mathrm{~A} * 03, \mathrm{~A} * 23, \mathrm{~B} * 14, \mathrm{~B} * 51, \mathrm{DRB} 1 * 01, \mathrm{DRB} 1 * 14$ & 98 & 96.67 & $\begin{array}{c}\text { A*03, A*23, A*30, B*49, B*14, B*51, B*35, } \\
\text { B*38, DRB } 1 * 01, \text { DRB } 1 * 04, \text { DRB } 1 * 14, \text { DRB } 1 * 03, \\
\text { DRB } 1 * 09, \text { DRB } 1 * 12, \text { DRB } 1 * 15\end{array}$ \\
\hline
\end{tabular}


Table 2. Cont.

\begin{tabular}{|c|c|c|c|c|}
\hline 51 & A2, A31(19), B18, B49(21), DR13(6), DR14(6) & 86 & 95 & $\begin{array}{c}A * 02, A * 31, A * 33, A * 23, B * 51, B * 52, B * 18, B * 15, \\
B * 40, B * 14, B * 55, \text { DRB } 1 * 04, \text { DRB } 1 * 11, \text { DRB } 1 * 12, \\
\text { DRB } 1 * 13, \text { DRB } 1 * 14, \text { DRB } 1 * 03\end{array}$ \\
\hline 52 & A2, A31, B18, B49, DR13, DR14 & Neg & 92 & $\begin{array}{l}\text { DRB } 1 * 08, \text { DRB } 1 * 11, \text { DRB } 1 * 12, \text { DRB } 1 * 13, \\
\text { DRB } 1 * 14, \text { DRB } 1 * 03, \text { DRB } 1 * 09, \text { DRB } 1 * 15\end{array}$ \\
\hline 53 & A3, A24, B38, B44, DRB1*04, DRB1*07 & 94 & 90 & DRB1*07, DRB1*04, DRB $1 * 15$ \\
\hline 54 & A25(10), A26, B18, B35, DR10, DR7 & Neg & 90 & DRB $1 * 01, \mathrm{DRB} 1 * 07, \mathrm{DRB} 1 * 09, \mathrm{DRB} 1 * 10$ \\
\hline 55 & A24(9), A31(19), B7, B13, DR4, DR53 & Neg & 80 & $\begin{array}{l}\text { DRB } 1 * 11, \text { DRB } 1 * 12, \text { DRB } 1 * 13, \text { DRB } 1 * 14 \\
\text { DRB } 1 * 15, \text { DRB } 1 * 16, \text { DRB } 1 * 03\end{array}$ \\
\hline 56 & $A * 11, A^{*} 26, \mathrm{~B} * 18, \mathrm{~B} * 55, \mathrm{DRB} 1 * 11, \mathrm{DRB} 1 * 13$ & Neg & 98 & $\begin{array}{c}\mathrm{DRB} 1 * 11, \mathrm{DRB} 1 * 12, \mathrm{DRB} 1 * 13, \mathrm{DRB} 1 * 03, \\
\mathrm{DRB} 1 * 01, \mathrm{DRB} 1 * 15\end{array}$ \\
\hline 57 & $\mathrm{~A} * 11 \mathrm{~A} * 26 \mathrm{~B} * 18 \mathrm{~B} * 55 \mathrm{DRB} 1 * 11 \mathrm{DRB} 1 * 13$ & 100 & Neg & $\mathrm{B} * 15, \mathrm{~B} * 35$ \\
\hline 58 & $\mathrm{~A} * 24, \mathrm{~A} * 30, \mathrm{~B} * 35, \mathrm{~B} * 53, \mathrm{DRB} 1 * 03, \mathrm{DRB} 1 * 11$ & 98 & $\mathrm{Neg}$ & $\mathrm{A} * 23, \mathrm{~B} * 44, \mathrm{~B} * 49, \mathrm{~B} * 51 / 52, \mathrm{~B} * 18, \mathrm{~B} * 15$ \\
\hline 59 & $A * 03, A^{*} 68, \mathrm{~B} * 07, \mathrm{~B} * 51, \mathrm{DRB} 1 * 07,-$ & Neg & 95 & DRB1*07, DRB1*12, DRB1*03, DRB $1 * 15$ \\
\hline 60 & $\mathrm{~A} 3, \mathrm{~A} 69, \mathrm{~B} 8, \mathrm{~B} 35, \mathrm{DRB} 1 * 03, \mathrm{DRB} 1 * 04$ & 92 & Neg & $A * 02, A * 03, A * 68, A * 34, A * 69, A * 33, A * 31$ \\
\hline
\end{tabular}

Table 3. The relation between unacceptable antigens in the patient group and population antigen frequencies

\begin{tabular}{lcccc}
\hline & Unacceptable antigens $(\boldsymbol{\%})$ & Population antigen $(\boldsymbol{\%})$ & $\boldsymbol{Z}$-value & $\boldsymbol{p}$ \\
\hline HLA-A*25 & 61.60 & 0.8 & 33.289 & $<0.001$ \\
\hline HLA-B*45 & 65 & 0.21 & 41.000 & $<0.001$ \\
\hline HLA-DRB1*04 & 41.6 & 14.45 & 5.833 & $<0.001$ \\
\hline DRB1*08 & 41.6 & 1.89 & 18.874 & $<0.001$ \\
\hline
\end{tabular}

program [16]. However, hypersensitive patients only with extra points in the standard Eurotransplant allocation program have about a $20 \%$ chance for transplantation within the same time period [14].

While the patients in the acceptable mismatch program will receive an organ more quickly, some studies suggest that graft survival of hypersensitive patients is lower than the other patients [17]. However, this is not the case for patients transplanted via the acceptable mismatch program. In recent studies, it was observed that the hypersensitized patients also have short term (two years) graft survival similar to the unsensitized patients [14]. In contrast, they revealed that other sensitized patients had indeed a significantly poorer graft survival. They suggested that graft survival of all sensitized patients could be increased by AM programs. Moreover, not only the short term but also the long term graft survival in patients transplanted from a suitable donor via the acceptable mismatch program was excellent $[16,18]$.

Recently, similar programs based on Eurotransplant acceptable mismatch program have been implemented in France, Italy, and Greece, whereas implementation is in progress in Scandia transplant, Switzerland, and Canada [18]. We expect that in the near future our country will implement a similar approach. The FC-SAB results of 60 patients waiting for transplantation will attribute to the pre-transplantation assessments and most probably the transplantation chance of these patients will be increased.

In conclusion, identification of SAB and the matching strategies that depend on both epitope sharing of mismatch antigens and related antibody production are very important to achieve transplantation. Thus, the transplantation chance of some patients may increase $25-50 \%$. If we look from the viewpoint of these results, we can prevent negative impacts on their psychology by avoiding the calls of the patients to each testing for transplantation from deceased donors.

The authors declare no conflict of interest.

The authors thank the staff of Tissue Typing Laboratory of Izmir Tepecik Education and Research Hospital for their continuous efforts in performing of the tests. This study was supported by Scientific Research Project Foundation of Izmir Katip Celebi University (Project No: 2013-1-TSBP-11). 


\section{References}

1. Vaidya S, Partlow D, Susskind B, et al. (2006): Prediction of crossmatch outcome of highly sensitized patients by single and/or multiple antigen bead luminex assay. Transplantation 82: $1524-1528$.

2. Doxiadis II, Duquesnoy RJ, Claas FH (2005): Extending options for highly sensitized patients to receive a suitable kidney graft. Curr Opin Immunol 17: 536-540.

3. Bray RA, Nolen JD, Larsen C, et al. (2006): Transplanting the highly sensitized patient: The emory algorithm. Am J Transplant 6: 2307-2315.

4. Patel R, Terasaki PI (1969): Significance of the positive crossmatch test in kidney transplantation. N Engl J Med 280: 735-739.

5. Lefaucheur C, Loupy A, Hill GS, et al. (2010): Preexisting donor-specific HLA antibodies predict outcome in kidney transplantation. J Am Soc Nephrol 21: 1398-1406.

6. Amico P, Hönger G, Mayr M, et al. (2009): Clinical relevance of pretransplant donor-specific HLA antibodies detected by single-antigen flow-beads. Transplantation 87: 1681-1688.

7. Gebel HM, Bray RA, Nickerson P (2003): Pre-transplant assessment of donor reactive, HLA-specific antibodies in renal transplantation: contraindication vs. risk. Am J Transplant 3: 1488-1500.

8. Pei R, Lee JH, Shih NJ, et al. (2003): Single human leukocyte antigen flow cytometry beads for accurate identification of human leukocyte antigen antibody specifities. Transplantation 75: 43-49.

9. Bielmann D, Hönger G, Lutz D, et al. (2007): Pretransplant risk assessment in renal allograft recipients using virtual crossmatching. Am J Transplant 7: 626-632.

10. Stegall MD, Gloor J, Winters JL, et al. (2006): A comparison of plasmapheresis versus high-dose IVIG desensitization in renal allograft recipients with high levels of donor specific alloantibody. Am J Transplant 6: 346-351.

11. Akalin E, Ames S, Sehgal V, et al. (2005): Intravenous immunoglobulin and thymoglobulin induction treatment in immunologically high-risk kidney transplant recipients. Transplantation 79: 742 .

12. Duquesnoy RJ, Witvliet M, Doxiadis II, et al. (2004): HLA Matchmaker-based strategy to identify acceptable HLA class I mismatches for highly sensitized kidney transplant candidates. Transpl Int 17: 22-30.

13. Dieplinger G, Ditt V, Arns W, et al. (2014): Luminex solid-phase assay after transplantation in a group of 88 consecutive living-donor renal transplantations. Transpl Int 27: 60-68.

14. Claas FH, Doxiadis II (2009): Management of the highly sensitized patient. Curr Opin Immunol 21: 569-572.

15. Gandhi MJ, Degoey S, Falbo D, et al. (2013): Inter and intra laboratory concordance of HLA antibody results obtained by single antigen bead based assay. Hum Immunol 74: 310-317.

16. Claas FH, Witvliet MD, Duquesnoy RJ, et al. (2004): The acceptable mismatch program as a fast tool for highly sensitized patients awaiting a cadaveric kidney transplantation: short waiting time and excellent graft survival. Transplantation 78: 190-193.

17. Jackson AM, Zachary AA (2008): The problem of transplanting the sensitized patients: whose problem is it? Front Biosci 13: 1396-1412.

18. Claas FH, Rahmel A, Doxiadis II (2009): Enhanced kidney allocation to highly sensitized patients by the acceptable mismatch program. Transplantation 88: 447-452. 